\title{
Investigation of the Negative Thermal Expansion of $\mathrm{ZrW}_{2} \mathrm{O}_{8}$
}

\author{
N. Ulbrich, W. Tröger, T. Butz, and P. Blaha ${ }^{a}$ \\ Nukleare Festkörperphysik, Fakultät für Physik und Geowissenschaften, Universität Leipzig, \\ Linnéstraße 5, D-04103 Leipzig \\ ${ }^{a}$ Institut für Technische Elektrochemie, Technische Universität Wien, Getreidemarkt 9/158, \\ A-1060 Wien
}

Reprint requests to Dr. W. T.; Fax: +49-341-97-32-729; E-mail: troeger@physik.uni-leipzig.de

Z. Naturforsch. 55a, 301-310 (2000); received October 28, 1999

Presented at the XVth International Symposium on Nuclear Quadrupole Interactions, Leipzig, Germany, July 25-30, 1999.

\begin{abstract}
The negative thermal expansion in $\mathrm{ZrW}_{2} \mathrm{O}_{8}$ was investigated on a microscopic scale by temperature dependent measurements of the electric field gradients at the nuclear probe ${ }^{187} \mathrm{~W}\left(\beta^{-}\right){ }^{187} \mathrm{Re}$ using time differential perturbed angular correlation spectroscopy. Two distinct nuclear quadrupole interactions $\left|V_{z=}^{\mathrm{Rel}}\right|=18.92(4) \cdot 10^{21} \mathrm{~V} / \mathrm{m}^{2}, \eta^{\mathrm{Rel}}=0.0$ and $\left|V_{z z}^{\mathrm{Re}}\right|=4.55(2) \cdot 10^{21} \mathrm{~V} / \mathrm{m}^{2}, \eta^{\mathrm{Re} 2}=0.053(3)$ were observed at $295 \mathrm{~K}$, which are assigned to the two crystallographically distinct $\mathrm{WO}_{4}$ tetrahedra of the room temperature structure. Ab initio calculations of electron densities and electric field gradients with $1: 7$ Re-impurities using the full potential linearized augmented plane wave package WIEN97 yield the electric field gradients $V_{z=}^{\mathrm{Re} 1}=12.63 \cdot 10^{21} \mathrm{~V} / \mathrm{m}^{2}, \eta^{\mathrm{Re} 1}=0.0$ and $V_{z z}^{\mathrm{Re} 2}=4.90 \cdot 10^{21} \mathrm{~V} / \mathrm{m}^{2}, \eta^{\mathrm{Re} 2}=0.0$. The observed temperature dependence of the nuclear quadrupole interactions agrees well with the structural phase transition at $428 \mathrm{~K}$ observed by neutron and x-ray diffraction. Our experiments corroborate the suggested mechanism of coupled librations of rigid $\mathrm{ZrO}_{6}$ octahedra and $\mathrm{WO}_{4}$ tetrahedra, which is an alternative description of transverse vibrations of oxygen atoms in $\mathrm{Zr}-\mathrm{O}-\mathrm{W}$ bonds, for the negative thermal expansion in $\mathrm{ZrW}_{2} \mathrm{O}_{8}$.
\end{abstract}

Key words: $\mathrm{ZrW}_{2} \mathrm{O}_{8}$; Negative Thermal Expansion; Phase Transition; Time Differential Perturbed Angular Correlation (TDPAC); Hyperfine Spectroscopy.

\section{Introduction}

Neutron and x-ray diffraction experiments revealed a strong negative thermal expansion in $\mathrm{ZrW}_{2} \mathrm{O}_{8}$ from $0.3 \mathrm{~K}$ up to its decomposition temperature at $1050 \mathrm{~K}$ [1]. The negative thermal expansion is isotropic and is not discontinuous at the structural phase transition at $428 \mathrm{~K}$. With an overall linear thermal expansion coefficient $\alpha=-8.7 \cdot 10^{-6} \mathrm{~K}^{-1}, \mathrm{ZrW}_{2} \mathrm{O}_{8}$ currently holds the record of intrinsic negative thermal expansion [2]. Only the isostructural compounds $\mathrm{Sc}_{2} \mathrm{~W}_{3} \mathrm{O}_{12}$ and $\mathrm{Lu}_{2} \mathrm{~W}_{3} \mathrm{O}_{12}$ appear to approach the degree of thermal contraction of $\mathrm{ZrW}_{2} \mathrm{O}_{8}$ with intrinsic linear coefficients of thermal expansion $\alpha=-2.2 \cdot 10^{-6} \mathrm{~K}^{-1}$ and $\alpha=-6.8 \cdot 10^{-6} \mathrm{~K}^{-1}$, respectively $[3,4]$.

This highly unusual thermal expansion property makes $\mathrm{ZrW}_{2} \mathrm{O}_{8}$ extremely well suited for potential applications both as a pure compound and as a component in composites with a tuneable overall thermal expansion. Applications include, e.g., substrates and heat sinks that match the thermal expansion of $\mathrm{Si}$, substrate materials with zero thermal expansion for mirrors in telescopes and satellites, and the adjustment of the thermal expansion of the white composites used in teeth fillings [5].

The origin of the negative thermal expansion in $\mathrm{ZrW}_{2} \mathrm{O}_{8}$ has been investigated both experimentally on a macroscopic scale using neutron and x-ray diffraction [6], and theoretically using lattice dynamics methods [7]. An explanation of the thermal contraction are transverse vibrations of the two-coordinated oxygen atoms in $\mathrm{Zr}$-O-W-bonds which contain strong $\mathrm{Zr}-\mathrm{O}$ and $\mathrm{W}-\mathrm{O}$ bonds showing negligible thermal expansion causing a reduction of the $\mathrm{Zr}$...W distance [6]. In a different approach, the negative thermal expansion of the low temperature phase of $\mathrm{ZrW}_{2} \mathrm{O}_{8}$ is attributed to coupled librations of the rigid $\mathrm{ZrO}_{6}$ octahedra and $\mathrm{WO}_{4}$ tetrahedra, the building blocks of an open framework structure [7]. Calculations based on this model yield a linear thermal expansion coefficient $\alpha=-9.4 \cdot 10^{-6} \mathrm{~K}^{-1}$. There is currently no evidence that these low-frequency phonon modes, which can apparently propagate without distortions of the polyhedral building blocks and are therefore called rigid unit modes, exist in the high temperature phase of $\mathrm{ZrW}_{2} \mathrm{O}_{8}$. The rigid unit mode model can be regarded as an alternative description of transverse vibrations of oxygen atoms in $\mathrm{Zr}-\mathrm{O}-\mathrm{W}$ bonds. 


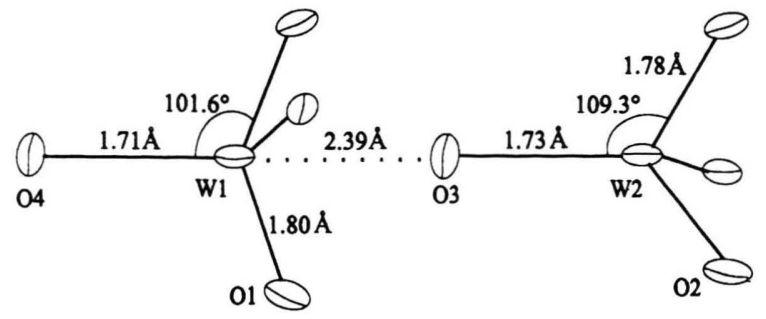

Fig. 1. The two crystallographically distinct $\mathrm{WO}_{4}$ tetrahedra of the room temperature structure arranged on the threefold rotation axis [6].

Recently, we started to investigate the nuclear quadrupole interaction (NQI) of ${ }^{187} \mathrm{~W}\left(\beta^{-}\right){ }^{187} \mathrm{Re}$ in $\mathrm{ZrW}_{2} \mathrm{O}_{8}$ at room temperature by time differential perturbed angular correlation (TDPAC) spectroscopy [8]. The present work reports on the temperature dependence of the electric field gradient (EFG) at substitutional W lattice sites using ${ }^{187} \mathrm{~W}\left(\beta^{-}\right){ }^{187} \mathrm{Re}$-TDPAC to shed light on the origin of the negative thermal expansion of in $\mathrm{ZrW}_{2} \mathrm{O}_{8}$ as well as on the structural phase transition at $428 \mathrm{~K}$ on a microscopic scale. Furthermore, ab initio electron density and electric field gradient calculations based on the density functional theory were carried out using the full potential linearized augmented plane wave (FPLAPW) package WIEN97 [9] to assign the measured EFGs to the lattice sites.

\section{Crystal Structure of $\mathrm{ZrW}_{2} \mathrm{O}_{8}$}

The structure of $\mathrm{ZrW}_{2} \mathrm{O}_{8}$ was determined by neutron and $\mathrm{x}$-ray diffraction experiments to be cubic over its entire existence range [6]. Below $428 \mathrm{~K}, \mathrm{ZrW}_{2} \mathrm{O}_{8}$ adopts the acentric space group $\mathrm{P}_{1} 3$ and has an ordered structure comprised of corner-sharing $\mathrm{ZrO}_{6}$ octahedra and two crystallographically distinct $\mathrm{WO}_{4}$ tetrahedra. The latter ones are arranged on a threefold rotation axis (see Figure 1).

Both tetrahedra differ from the shape of a perfect tetrahedron with the $\mathrm{W} 1$ tetrahedron being more distorted than the W2 tetrahedron. Each oxygen atom of a $\mathrm{ZrO}_{6}$ octahedron is shared with a $\mathrm{WO}_{4}$ tetrahedron, whereas each $\mathrm{WO}_{4}$ tetrahedron shares only three oxygen atoms with a $\mathrm{ZrO}_{6}$ octahedron (O1 and $\mathrm{O} 2$ in Figure 1). The remaining oxygen atom on the threefold rotation axis (O3 and $\mathrm{O} 4$ in Figure 1) is only one-coordinated forming a terminal W-O bond.

Above the phase transition at $428 \mathrm{~K}$, which is supposed to be second order, the space group becomes centric $\mathrm{Pa} \overline{3}$. The observed increase of the ionic conductivity in the high temperature phase is attributed to the high oxygen (a)

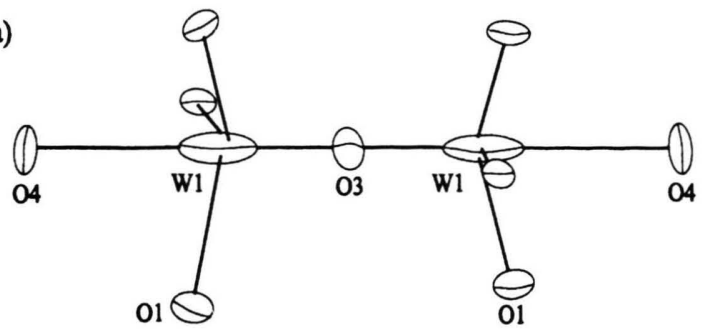

(b)

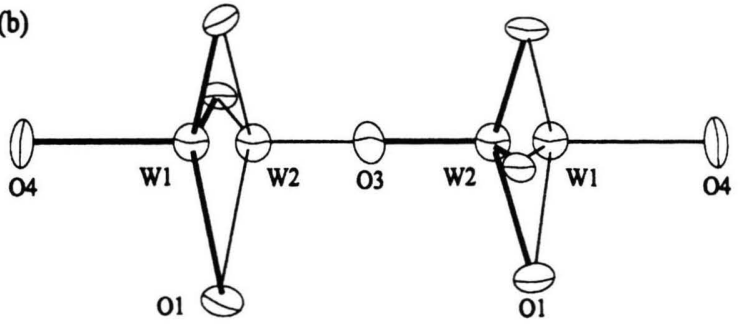

Fig. 2. The two $\mathrm{WO}_{4}$ tetrahedra of the high temperature structure: (a) model with a single W site; (b) model with disordered $\mathrm{W}$ and $\mathrm{O}$ sites [6].

mobility at elevated temperatures [6]. The structure is now disordered giving rise to two different structural refinement models. The first model proposed in [6] suggests a single $\mathrm{W}$ site with considerable disorder of $\mathrm{W}$ atoms along the threefold rotation axis (see Figure $2 \mathrm{a}$ ).

A better structural refinement was obtained with a model containing disordered $\mathrm{W}$ and $\mathrm{O}$ sites on the threefold axis (see Figure 2b). This model contains two partially occupied configurations indicated in Fig. 2 b by either thick or thin lines. The two configurations can be obtained by reflecting a single tetrahedron through the plane of the three bridging oxygen atoms $\mathrm{Ol}$. Such a rearrangement of the terminal W-O bond involves correlated reorientations of the neighbouring $\mathrm{WO}_{4}$ tetrahedra along the three-fold axis in order to avoid unacceptably close $\mathrm{O} \ldots \mathrm{O}$ distances at the $\mathrm{O} 3$ position. These correlated reflections lead to an alternating occupation of the two oxygen sites $\mathrm{O} 4$ on the threefold rotation axis requiring a high oxygen mobility in the high temperature phase. In summary, $\mathrm{ZrW}_{2} \mathrm{O}_{8}$ undergoes a very unusual order-disorder phase transition upon heating.

\section{Experimental}

\subsection{Method}

The TDPAC measurements were carried out using the $497.5-72.0 \mathrm{keV} \gamma-\gamma$-cascade of the TDPAC probe ${ }^{187} \mathrm{~W}$ 
$\left(\beta^{-}\right)^{187} \mathrm{Re}$. The isotope ${ }^{187} \mathrm{~W}$ (half-life $\tau_{1 / 2}=23.72 \mathrm{~h}$ ) is produced by thermal neutron capture of ${ }^{186} \mathrm{~W}$ which has a natural isotopic abundance of $28.6 \%$ and a thermal neutron cross section of $37.7 \mathrm{~b}$. The $I=9 / 2$ intermediate state of ${ }^{187} \mathrm{Re}$ has a half-life of $\tau_{1 / 2}=555.3 \mathrm{~ns}$ and a large nuclear quadrupole moment of $Q=3.04$ (5) b which may lead to high NQI frequencies. Although the anisotropy $A_{22}=-13.1 \%$ [10] of the $\gamma-\gamma$-cascade is high, an admixture of prompt x-rays in the $72.0 \mathrm{keV}$ stop-channel reduces drastically the effective anisotropy to $A_{22}^{\text {eff }} \simeq-2 \%$ $[8,11]$. Therefore, the measurements were carried out using two extremely efficient six detector TDPAC spectrometers as described in [12]. One spectrometer was equipped with conically capped $\mathrm{BaF}_{2}$-scintillation detectors with an excellent time resolution for the detection of high nuclear precession frequencies. The full width at half maximum (FWHM) value of the time resolution function was $\tau_{\mathrm{FWHM}}=0.8 \mathrm{~ns}$ for the $497.5-72.0 \mathrm{keV}$ $\gamma-\gamma$-cascade, the FWHM value of the relative energy resolution for the $662 \mathrm{keV} \gamma$-line of ${ }^{137} \mathrm{Cs}$ was $15 \%$. The second spectrometer was equipped with cylindrical $\mathrm{NaI}(\mathrm{Tl})$-scintillation detectors in order to obtain a better energy discrimination at $72.0 \mathrm{keV}$ leading to a slightly improved effective anisotropy $A_{22}^{\text {eff }} \simeq-3 \%$. Here, the time resolution was $\tau_{\mathrm{FWHM}}=3.5 \mathrm{~ns}$ and the relative energy resolution was 7\% (FWHM) at $662 \mathrm{keV}$.

Taking the two distinct $W$ lattice sites into account, the TDPAC time spectra $A_{22} G_{22}(t)$ were fitted using the powder perturbation function

$$
\begin{aligned}
G_{22}(t)= & \sum_{i=1}^{2} \operatorname{amp}^{(i)} \sum_{n=0}^{10} a_{n}^{(i)}\left(\eta^{(i)}\right) \\
& \cdot \exp \left(-0.09 \tau_{\mathrm{FWHM}}^{2}\left(\omega_{n}^{(i)}\left(\eta^{(i)}\right)\right)^{2}\right) \\
& \cdot \exp \left(-\delta^{(i)} \omega_{n}^{(i)}\left(\eta^{(i)}\right) \cdot t\right) \\
& \cdot \cos \left(\omega_{n}^{(i)}\left(\eta^{(i)}\right) \cdot t\right)+\text { const }
\end{aligned}
$$

where $\operatorname{amp}^{(i)}$ is the time independent amplitude at site $i$, const. is a time independent base-line, $\tau_{\mathrm{FWHM}}$ is the FWHM value of the time resolution function and $\delta^{(i)}$ the half width at half maximum value of the inhomogeneous Lorentzian line broadening at site $i$. The coefficients $a_{n}^{(i)}$ $\left(\eta^{(i)}\right)$ and the precession frequencies $\omega_{n}^{(i)}\left(\eta^{(i)}\right)$ are calculated for a nuclear spin $I=9 / 2$ and are functions of the asymmetry parameter $\eta^{(i)}$ [13].

\subsection{Sample Preparation}

$\mathrm{ZrW}_{2} \mathrm{O}_{8}$ was synthesised from its constituent oxides $\mathrm{ZrO}_{2}$ and $\mathrm{WO}_{3}$ by Sleight and co-workers [14]. Powder samples of $5 \mathrm{mg} \mathrm{ZrW} \mathrm{ZO}_{8}$ of natural isotopic abundance were irradiated either for $2 \mathrm{~h}$ in a thermal neutron flux of $2 \cdot 10^{13} \mathrm{~cm}^{-2} \mathrm{~s}^{-1}$ at the Forschungsreaktor München FRM I or for $4 \mathrm{~h}$ in a thermal neutron flux of $1 \cdot 10^{13}$ $\mathrm{cm}^{-2} \mathrm{~s}^{-1}$ at the Forschungsreaktor BER II at the HahnMeitner-Institut Berlin. All TDPAC-measurements were performed without annealing the samples, since annealing at $600 \mathrm{~K}$ under a continuous oxygen flow and subsequent measurements at $295 \mathrm{~K}$ did not produce any noticeable effect compared to previous measurements without annealing. All measurements above room temperature were performed in a specially designed furnace as described in [15] under a continuous flow of oxygen at ambient pressure.

\section{Experimental Results}

The TDPAC time spectra were recorded at the temperatures $17 \mathrm{~K}, 77 \mathrm{~K}, 150 \mathrm{~K}, 243 \mathrm{~K}, 295 \mathrm{~K}, 334 \mathrm{~K}, 373 \mathrm{~K}$, and $403 \mathrm{~K}$, i.e. below the phase transition at $428 \mathrm{~K}$, and at $503 \mathrm{~K}$ in the high temperature phase. On average we collected $530 \cdot 10^{6}$ coincidences per spectrum with an average data collection time of five weeks per spectrum using several samples sequentially. Figures 3 , 4, and 7 show the TDPAC time spectra $A_{22} G_{22}(t)$ and their cosine transforms $I(\omega)$. For the sake of clarity in the data presentation the number of channels of the time spectra was compressed from 800 to 200 with a corresponding fourfold increase in the time per channel. The spectra were recorded with either the $\mathrm{BaF}_{2^{-}}$or the $\mathrm{NaI}(\mathrm{Tl})$ TDPAC spectrometer. At some temperatures the measurements were performed with both the $\mathrm{BaF}_{2}$ - and the $\mathrm{NaI}(\mathrm{Tl})$-TDPAC-spectrometers, and the data were added in order to improve the statistical accuracy of the data. Table 1 shows the temperature dependent NQI parameters of the observed NQIs at W1 and W2 sites as obtained by a least squares fit analysis of the TDPAC time spectra. The temperature dependence of the NQI parameters $v_{\mathrm{Q}}^{(1)}, v_{\mathrm{Q}}^{(2)}, \eta^{(2)}, \delta^{(1)}$, and $\delta^{(2)}$ is plotted in Figs. 8, 9, and 11 , respectively.

At every temperature below the phase transition we observed two distinct NQI signals with characteristic nuclear precession frequencies $\omega^{(1)}$ and $\omega^{(2)}$. The obtained NQI parameters $\omega^{(1)}=364.1(3) \mathrm{Mrad} / \mathrm{s}$, $\eta^{(1)}=0.0$ and $\omega^{(2)}=89.7$ (2) Mrad/s, $\eta^{(2)}=0.053$ (3) for the $295 \mathrm{~K}$ spectrum are in perfect agreement with our previously reported room temperature data $\omega^{(1)}=363.7(4) \mathrm{Mrad} / \mathrm{s}, \quad \eta^{(1)}=0.0$ and $\omega^{(2)}=89.9(3)$ $\mathrm{Mrad} / \mathrm{s}, \eta^{(2)}=0.056(4)[8]$. 

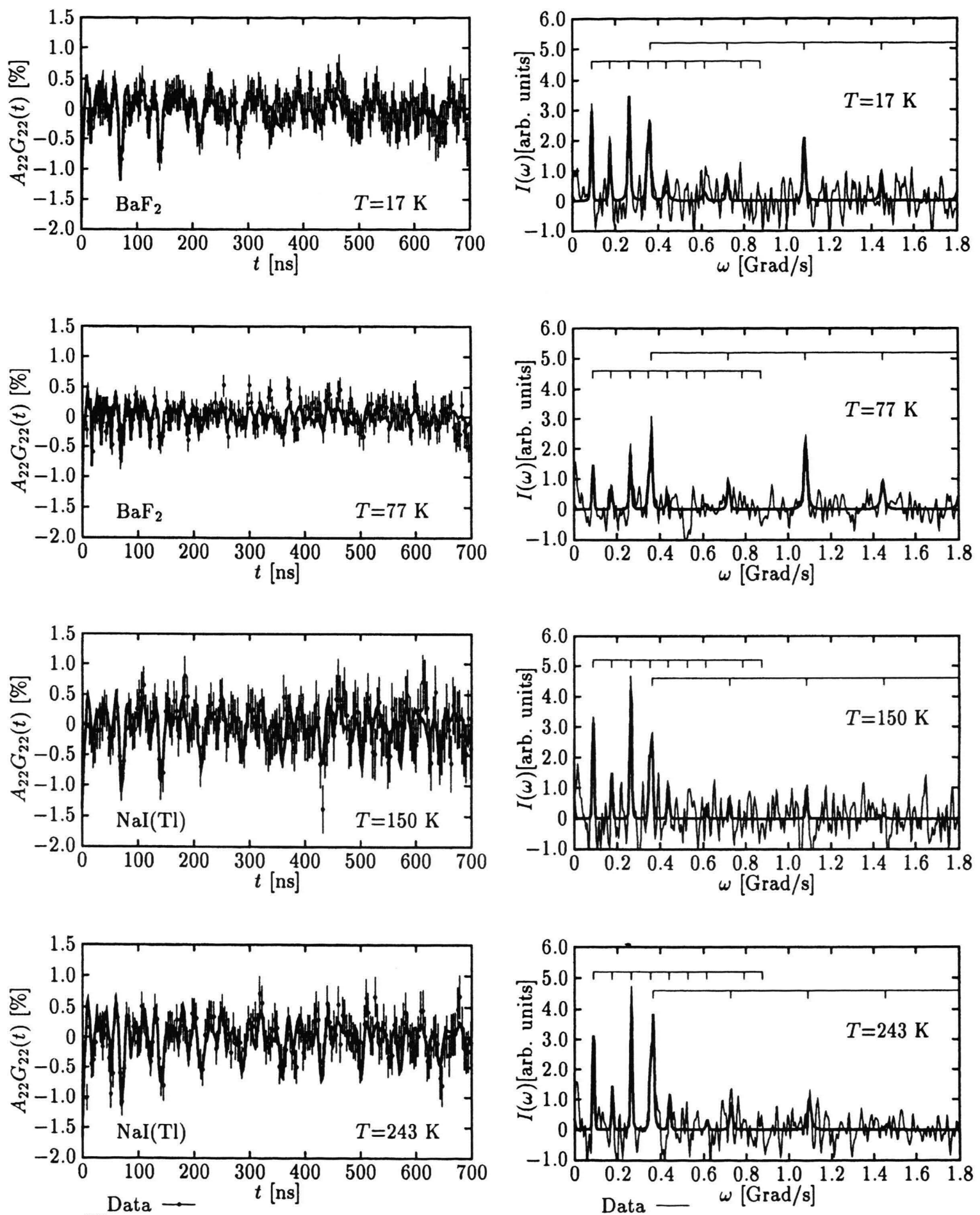

Theory —

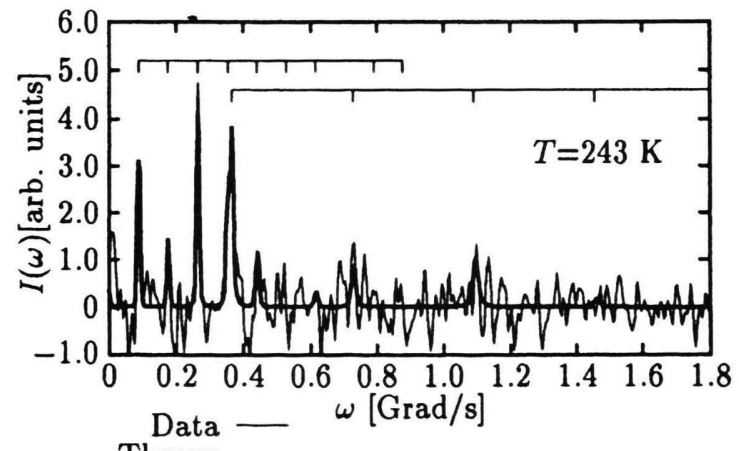

Fig. 3. TDPAC time spectra $A_{22} G_{22}(t)$ (left) and their cosine transforms $I(\omega)$ (right) at $17 \mathrm{~K}, 77 \mathrm{~K}, 150 \mathrm{~K}$ and $243 \mathrm{~K}$. The spectra were recorded with either the $\mathrm{BaF}_{2}$ - or the $\mathrm{NaI}(\mathrm{Tl})$-TDPAC-spectrometer. Bars in the cosine transformed spectra correspond to line positions for an $I=9 / 2$ split state. 

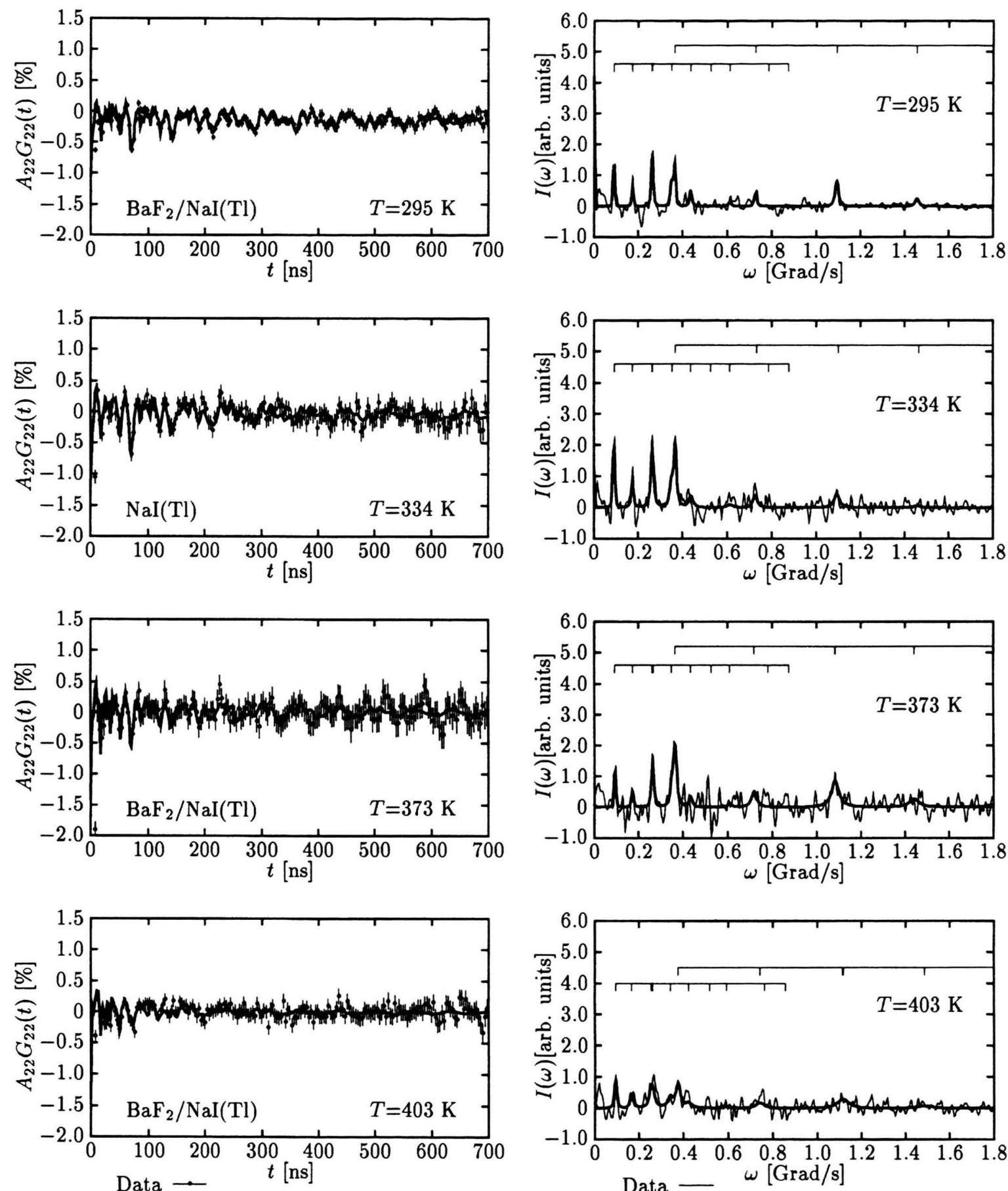

Theory -

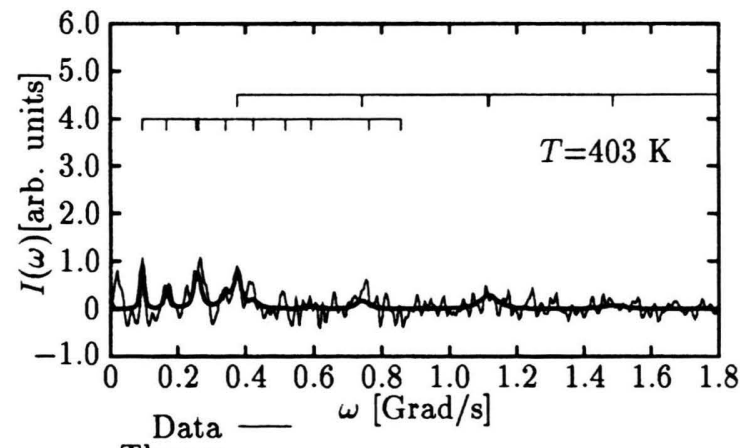

Fig. 4. TDPAC time spectra $A_{22} G_{22}(t)$ (left) and their cosine transforms $I(\omega)$ (right) at $295 \mathrm{~K}, 334 \mathrm{~K}, 373 \mathrm{~K}$ and $403 \mathrm{~K}$. The spectrum at $334 \mathrm{~K}$ was recorded with the $\mathrm{NaI}(\mathrm{Tl})$ - $\mathrm{TDPAC}$-spectrometer, the other spectra with both the $\mathrm{NaI}(\mathrm{Tl})-$ and the $\mathrm{BaF}_{2-}$ TDPAC-spectrometers. Bars in the cosine transformed spectra correspond to line positions for an $I=9 / 2$ split state. 

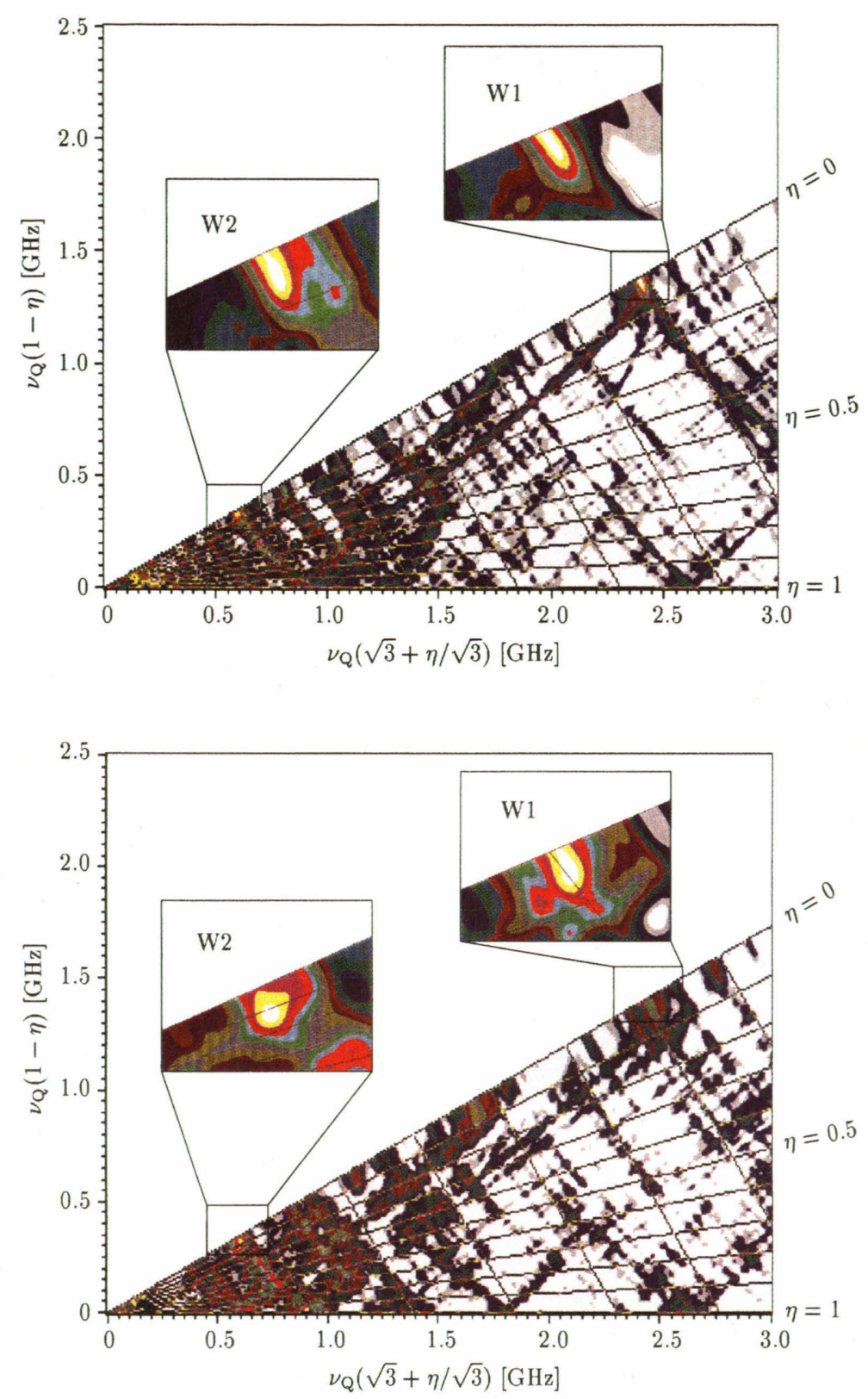

Fig. 5. Czizek-plot of the NQI at ${ }^{187} \mathrm{~W}\left(\beta^{-}\right)^{187} \mathrm{Re}$ in $\mathrm{ZrW}_{2} \mathrm{O}_{8}$ at $77 \mathrm{~K}$.
Fig. 6. Czizek-plot of the NQI at ${ }^{187} \mathrm{~W}\left(\beta^{-}\right)^{187} \mathrm{Re}$ in $\mathrm{ZrW}_{2} \mathrm{O}_{8}$ at $403 \mathrm{~K}$.
The amplitudes amp ${ }^{(1)}$ of the high frequency site tend to be somewhat bigger than the amplitudes amp ${ }^{(2)}$ of the low frequency site. Since the extremely good statistical quality of the room temperature data published in [8] $\left(1500 \cdot 10^{6}\right.$ coincidences with $\mathrm{BaF}_{2}$-detectors and 800 . $10^{6}$ coincidences with $\mathrm{NaI}(\mathrm{Tl})$-detectors) and the $295 \mathrm{~K}$ data of the present work show that the two NQI signals are equally populated within experimental error, we as- sume this to be true at the other temperatures, too, in spite of the fact that the freely adjusted amplitudes exhibit considerable variations (see Table 1). We assign the high frequency NQI $\left(\omega^{(1)}, \eta^{(1)}\right)$ to the strongly distorted W1 tetrahedron (site $i=1)$ and the low frequency NQI $\left(\omega^{(2)}\right.$, $\eta^{(2)}$ ) to the less distorted W2 tetrahedron (site $i=2$ ). This assignment is corroborated by our ab initio EFG calculations as described in Section 5. 
Table 1. Temperature dependent parameters of the observed NQIs at ${ }^{187} \mathrm{~W}\left(\beta^{-}\right)^{187} \mathrm{Re}$ in $\mathrm{ZrW}_{2} \mathrm{O}_{8}$.

\begin{tabular}{|c|c|c|c|c|c|c|}
\hline $\begin{array}{l}T \\
{[\mathrm{~K}]}\end{array}$ & $\begin{array}{l}a m p^{(1)} \\
{[\%]}\end{array}$ & $\begin{array}{l}\delta^{(1)} \\
{[\%]}\end{array}$ & $\begin{array}{l}\omega^{(1)} \\
{[\mathrm{Mrad} / \mathrm{s}]}\end{array}$ & $\eta^{(1)}$ & $\begin{array}{l}v_{\mathrm{Q}}^{(1)} \\
{[\mathrm{MHz}]}\end{array}$ & $\begin{array}{l}\left|V_{z z}^{(1)}\right| \\
{\left[10^{21} \mathrm{~V} \mid\right.} \\
\left.\mathrm{m}^{2}\right]\end{array}$ \\
\hline $\begin{array}{r}17 \\
77 \\
150 \\
243 \\
295 \\
334 \\
373 \\
403\end{array}$ & $\begin{array}{l}-0.9(1) \\
-1.3(1) \\
-1.2(3) \\
-2.4(2) \\
-0.8(1) \\
-1.6(2) \\
-1.8(1) \\
-1.0(3)\end{array}$ & $\begin{array}{l}0.3(1) \\
0.6(1) \\
0.1(2) \\
0.6(1) \\
0.6(1) \\
1.2(3) \\
1.7(3) \\
2.7(9)\end{array}$ & $\begin{array}{l}361.3(4) \\
361.3(4) \\
362.3(5) \\
363.8(6) \\
364.1(3) \\
367.1(9) \\
364.1(1) \\
376 \quad(3)\end{array}$ & $\begin{array}{l}0.0 \\
0.0 \\
0.0 \\
0.0 \\
0.0 \\
0.031(8) \\
0.03(1) \\
0.03 \quad(2)\end{array}$ & $\begin{array}{l}1380(3) \\
1380(3) \\
1384(3) \\
1390(4) \\
1391(3) \\
1389(7) \\
1376(9) \\
1421(24)\end{array}$ & $\begin{array}{l}18.78(4) \\
18.78(4) \\
18.83(5) \\
18.90(5) \\
18.92(4) \\
18.9(1) \\
18.7(1) \\
19.3(3)\end{array}$ \\
\hline $\begin{array}{l}T \\
{[\mathrm{~K}]}\end{array}$ & $\begin{array}{l}\mathrm{amp}^{(2)} \\
{[\%]}\end{array}$ & $\begin{array}{l}\delta^{(2)} \\
{[\%]}\end{array}$ & $\begin{array}{l}\omega^{(2)} \\
{[\mathrm{Mrad} / \mathrm{s}]}\end{array}$ & & $\begin{array}{l}v_{\mathrm{Q}}^{(2)} \\
{[\mathrm{MHz}]}\end{array}$ & $\begin{array}{l}\left|V_{z z}^{(2)}\right| \\
{\left[10^{21} \mathrm{~V} /\right.} \\
\left.\mathrm{m}^{2}\right]\end{array}$ \\
\hline $\begin{array}{r}17 \\
77 \\
150 \\
243 \\
295 \\
334 \\
373 \\
403\end{array}$ & $\begin{array}{l}-1.4(1) \\
-0.6(1) \\
-1.4(2) \\
-1.3(1) \\
-0.6(1) \\
-1.1(1) \\
-0.6(1) \\
-0.6(1)\end{array}$ & $\begin{array}{l}1.2(3) \\
0.7(3) \\
0.4(2) \\
0.4(1) \\
1.0(1) \\
2.0(4) \\
1.3(6) \\
4 \quad(1)\end{array}$ & $\begin{array}{l}89.4(4) \\
89.6(6) \\
89.1(4) \\
88.6(3) \\
89.7(2) \\
90.5(4) \\
93.3(7) \\
94 \quad(1)\end{array}$ & $\begin{array}{l}0.045(7) \\
0.053(7) \\
0.043(6) \\
0.031(6) \\
0.053(3) \\
0.060(5) \\
0.090(7) \\
0.114(9)\end{array}$ & $\begin{array}{l}335(2) \\
334(2) \\
335(2) \\
335(2) \\
334(1) \\
335(2) \\
333(3) \\
325(5)\end{array}$ & $\begin{array}{l}4.56(3) \\
4.54(3) \\
4.56(2) \\
4.56(2) \\
4.55(2) \\
4.56(3) \\
4.53(4) \\
4.42(7)\end{array}$ \\
\hline
\end{tabular}

Between $17 \mathrm{~K}$ and $295 \mathrm{~K}$ we observed axial symmetry at the more distorted $\mathrm{W} 1$ tetrahedron $\left(\eta^{(1)}=0.0\right)$. Only near the phase transition at $334 \mathrm{~K}, 373 \mathrm{~K}$ and $403 \mathrm{~K}$ we observed a non-vanishing asymmetry parameter $\eta^{(1)}=0.031(8), \eta^{(1)}=0.03(1)$ and $\eta^{(1)}=0.0 .03(2)$, respectively. At the less distorted W2 tetrahedron we observed a non-vanishing asymmetry parameter $\eta^{(2)}$ throughout the entire investigated temperature range of the room temperature phase.

The zero and the small non-zero asymmetry parameters can be best visualized by a cross-correlation analysis of the experimental cosine transform $I(\omega)$ with a theoretical spectrum for all possible values of $v_{\mathrm{Q}}$ and $\eta[16]$. The results are displayed in the contour plots of Fig. 5 and 6 for the spectra at $77 \mathrm{~K}$ and $403 \mathrm{~K}$. In these so-called Czjzek-plots [17] lines of constant $v_{\mathrm{Q}}$ are perpendicular to the upper boundary of the contour plot. Lines of constant $\eta$ are straight lines ranging from the upper boundary ( $\eta=0$ line) down to the horizontal ( $\eta=1$ line $)$. The insets are the zoomed regions of the $\left(v_{\mathrm{Q}}, \eta\right)$ spots of the $\mathrm{W} 1$ and the $\mathrm{W} 2$ site. The $\left(v_{\mathrm{Q}}, \eta\right) \operatorname{spot}^{1}$ of the W1 site at $77 \mathrm{~K}$ (see Fig. 5) has its maximum intensity at the $\eta=0$

\footnotetext{
I Actually, only half a spot is displayed, and the elongated shape is a consequence of the quadratic dependence of the eigenvalues of the Hamiltonian on $\eta$ for small $\eta$ and half-integer spins.
}
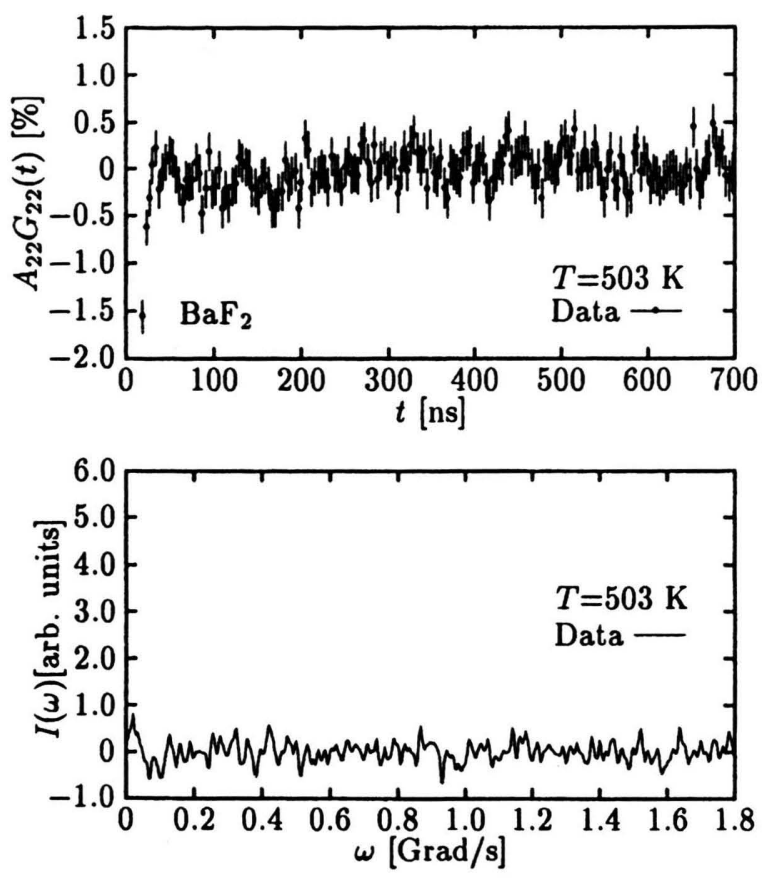

Fig. 7. Time spectrum $A_{22} G_{22}(t)$ (top) and its cosine transform $I(\omega)$ (bottom) at $503 \mathrm{~K}$.

line, i.e. an asymmetry parameter $\eta^{(1)}=0$, whereas the signal of the W2 site has its maximum intensity slightly off the $\eta=0$ line, i.e. a non-vanishing asymmetry parameter $\eta^{(2)}$. At $403 \mathrm{~K}$ near the phase transition (see Fig. 6) both signals have their maximum intensity significantly off the $\eta=0$ line, i.e. non-vanishing asymmetry parameters $\eta^{(1)}$ and $\eta^{(2)}$. No characteristic NQI signal was observable at $503 \mathrm{~K}$ of the high temperature phase of $\mathrm{ZrW}_{2} \mathrm{O}_{8}$ (see Figure 7).

The temperature dependence of the quadrupole coupling constants $v_{\mathrm{Q}}^{(1)}$ and $v_{\mathrm{Q}}^{(2)}$ of the $\mathrm{W} 1$ and $\mathrm{W} 2$ sites in $\mathrm{ZrW}_{2} \mathrm{O}_{8}$ is shown in Figure 8. $v_{\mathrm{Q}}^{(1)}(T)$ increases only slightly by $0.6 \%$ between $17 \mathrm{~K}$ and $334 \mathrm{~K}$ while $v_{\mathrm{Q}}^{(2)}(T)$ remains constant within the error limits in the respective temperature range. The asymmetry parameter $\eta^{(1)}$ at the W1 site remains zero up to $295 \mathrm{~K}$ and increases near the phase transition at $334 \mathrm{~K}, 373 \mathrm{~K}$ and $403 \mathrm{~K}$, whereas the temperature dependence of the asymmetry parameter $\eta^{(2)}$ at the W2 site (see Fig. 9) appears to parallel the thermal variation of the terminal W2-O3 bond length of the W2 tetrahedron as reported in [6] and shown in Figure 10. For comparison, Fig. 10 contains also the thermal variation of the terminal W1-O4 bond length of the W1 tetrahedron [6]. The inhomogeneous line broadening $\delta^{(1)}$ of 

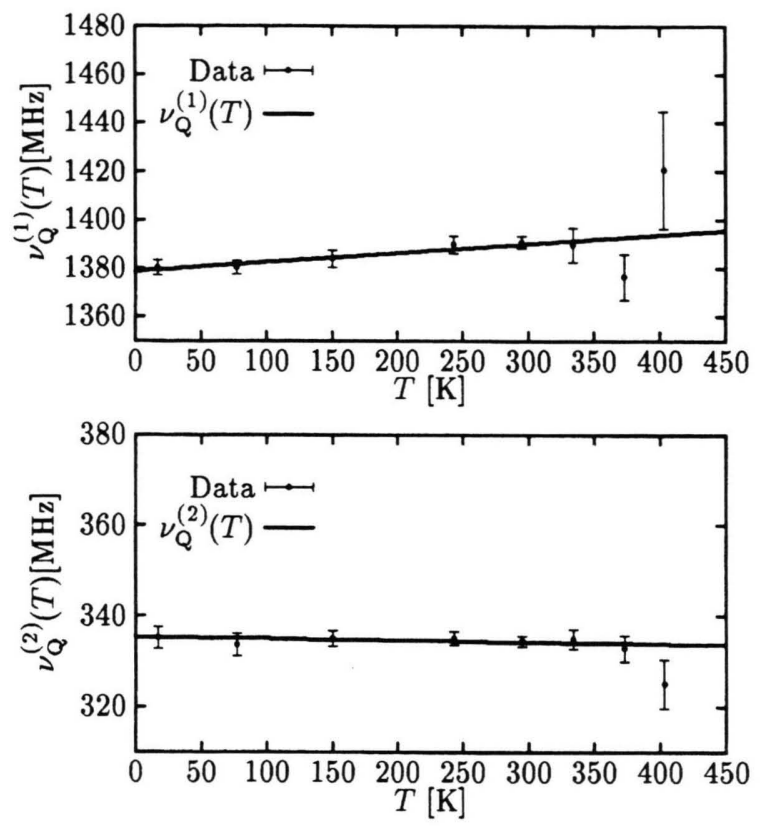

Fig. 8. Temperature dependence $v_{\mathrm{Q}}^{(1)}(T)$ (top) and $v_{\mathrm{Q}}^{(2)}(T)$ (bottom) at the W1 and W2 site.

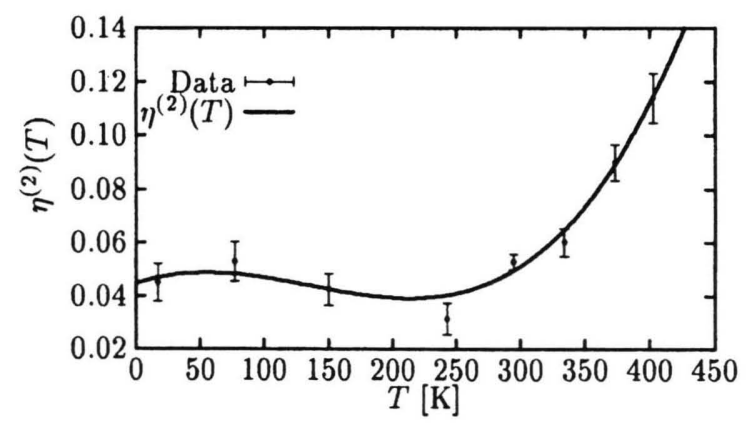

Fig. 9. Temperature dependence of the asymmetry parameter $\eta^{(2)}$ at the W2 site.

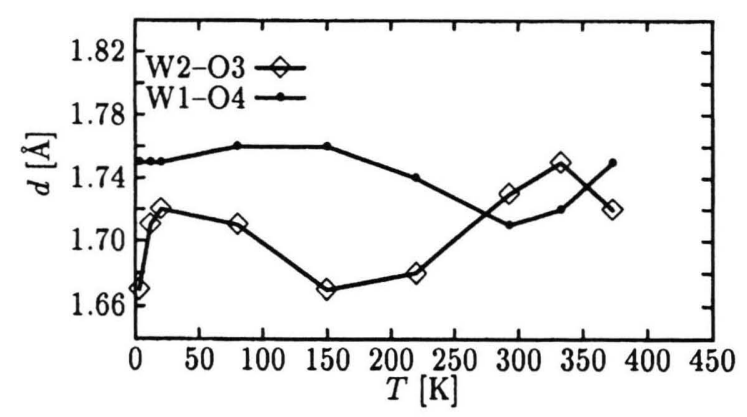

Fig. 10. Temperature dependence of the W1-O4 and the W2-O3 bond length as reported in [6].
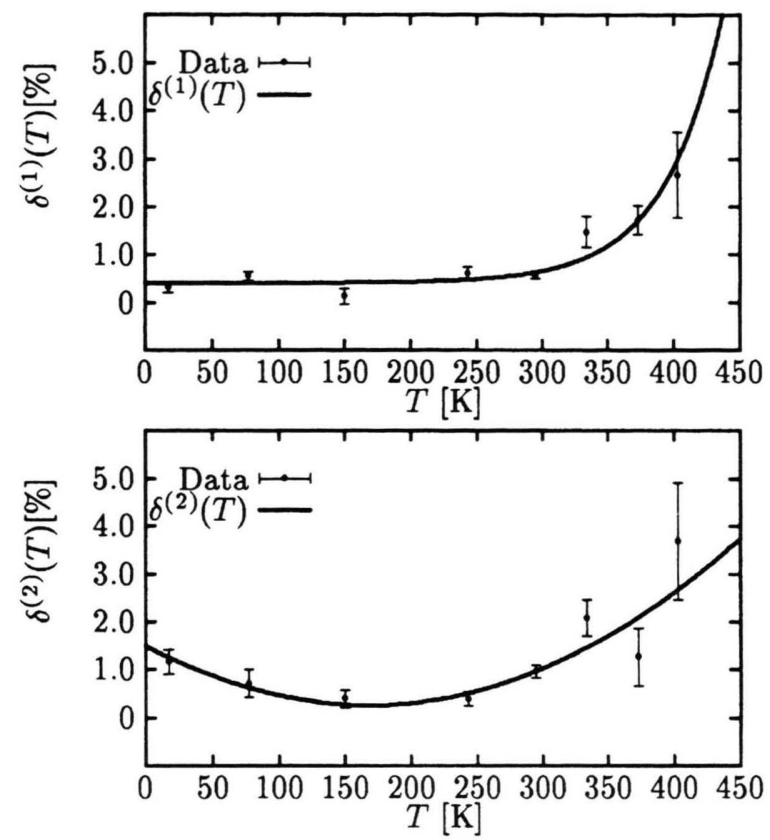

Fig. 11. Temperature dependence $\delta^{(1)}(T)$ (top) and $\delta^{(2)}(T)$ (bottom) of the inhomogeneous line broadening at the W1 and the W2 site.

the W1 site (see Fig. 11, top) shows only a small increase of $0.8 \%$ from $17 \mathrm{~K}$ up to $295 \mathrm{~K}$ and then increases to about $3 \%$ at $403 \mathrm{~K}$. At the W2 site (see Fig. 11, bottom) $\delta^{(2)}$ first decreases slightly and then goes up to about $4 \%$ at $403 \mathrm{~K}$.

\section{Electric Field Gradient Calculations}

Ab initio calculations of electron densities and electric field gradients in $\mathrm{ZrW}_{2} \mathrm{O}_{8}$ and $\mathrm{ZrW}_{2} \mathrm{O}_{8}$ : Re were carried out using the FPLAPW package WIEN97 in order to assign the observed NQIs at ${ }^{187} \mathrm{~W}\left(\beta^{-}\right){ }^{187} \mathrm{Re}$ to the corresponding lattice sites. No supercell calculations were possible due to the large number of 44 atoms per unit cell. Therefore, the Re impurities were introduced by replacing one of the eight $\mathrm{W}$ atoms contained in the unit cell by $\operatorname{Re}$, i.e. $\operatorname{Re}: W=1: 7$. The following three types of calculations were carried out:

(i) pure compound

(ii) impurity system with $\mathrm{Re}$ at $\mathrm{W} 1$ site

(iii) impurity system with $\mathrm{Re}$ at $\mathrm{W} 2$ site.

The fractional atomic coordinates in space group $\mathrm{P} 2{ }_{1} 3$ used for both the pure compound and the impurity system 
Table 2. Electric field gradient $V_{\tilde{-}}^{(i)}$, asymmetry parameter $\eta^{(i)}$ and absolute value of the force $\left|\tilde{F}^{(i)}\right|$ on the atom $i$ as calculated in the pure compound.

\begin{tabular}{lcll}
\hline Atom $i$ & $V_{z i}^{(i)}\left[10^{21} \mathrm{~V} / \mathrm{m}^{2}\right]$ & $\eta^{(i)}$ & $\left|F^{(i)}\right|[\mathrm{mRy} /$ a.u. $]$ \\
\hline Zr & 2.45 & 0.0 & 13.5 \\
W1 & 12.27 & 0.0 & 23.9 \\
W2 & 1.65 & 0.0 & 4.0 \\
O1 & -2.65 & 0.741 & 37.2 \\
O2 & 2.07 & 0.353 & 28.4 \\
O3 & 1.19 & 0.0 & 29.2 \\
O4 & -3.46 & 0.0 & 44.9 \\
\hline
\end{tabular}

Table 3. Calculated electric field gradient $V_{\tilde{z}}^{(i)}$, asymmetry parameter $\eta^{(i)}$ and absolute value of the force $\left|F^{(i)}\right|$ on Rel and $\mathrm{Re} 2$ in the respective impurity system.

\begin{tabular}{llll}
\hline Atom $i$ & $V_{\tilde{z}}^{(i)}\left[10^{21} \mathrm{~V} / \mathrm{m}^{2}\right]$ & $\eta^{(i)}$ & $\left|F^{(i)}\right|[\mathrm{mRy} /$ a.u. $]$ \\
\hline Re1 & 12.63 & 0.0 & 49.4 \\
$\operatorname{Re} 2$ & 4.90 & 0.0 & 0.5 \\
\hline
\end{tabular}

can be found in [1]. The cubic cell edge was $a=9.15993(5) \AA$ at $293 \mathrm{~K}$.

Full convergence of the plane wave basis set could be obtained with $20 \boldsymbol{k}$-points in the irreducible wedge of the Brillouin zone. Convergence tests were carried out using convergence parameters $\mathrm{RKM}=4.0, \mathrm{RKM}=5.0$ and $\mathrm{RKM}=6.0$ [18]. Due to main memory limitations the convergence parameter RKM was restricted to RKM = 6.0. The size of the subprogram LAPW1C required to achieve RKM $=6.0$ was $961.1 \mathrm{MB}$. The CPU time per iteration was about $354 \mathrm{~h}$ on a SPP2000/X-48 computer. Table 2 shows the results of the calculations for $V_{z z}^{(i)}$ and $\eta^{(i)}$ at the position of each inequivalent atom $i$ of the pure compound. The absolute value of the force $\left|F^{(i)}\right|$ on the atom $i$ is also listed in Table 2. Table 3 shows the results for $V_{z z}^{\mathrm{Re} 1}, \eta^{\mathrm{Re} 1}$, and $\left|F^{\mathrm{Re} 1}\right|$ of the Rel-impurity system and for $V_{z z}^{\mathrm{Re} 2}, \eta^{\mathrm{Re} 2}$, and $\left|F^{\mathrm{Re} 2}\right|$ of the Re2-impurity system. With the exception of the W2 and Re2 sites, the large forces of the order of $10 \mathrm{mRy} / \mathrm{a} . \mathrm{u}$. in both the pure compound and the impurity system indicate that a structural relaxation is necessary or that the numerical accuracy has to be improved by using a higher convergence parameter RKM. The direction of the force $F^{\mathrm{Rel}}$ is such that relaxations would lead to a smaller EFG for Re1, which was verified by corresponding calculations. Due to the excessive computing time requirements and main memory limitations no further calculations were carried
Table 4. Comparison of the experimental values $\left|V_{z z}^{\mathrm{Rel}}\right|, \eta^{\mathrm{Re} 1}$ and $\left|V_{\tilde{z}}^{\mathrm{Re} 2}\right|, \eta^{\mathrm{Re} 2}$ at $T=295 \mathrm{~K}$ with the calculated values of the impurity systems.

\begin{tabular}{|c|c|c|c|c|}
\hline \multirow[t]{2}{*}{ Atom $i$} & \multicolumn{2}{|c|}{ Experiment (TDPAC) } & \multicolumn{2}{|c|}{ Theory (FPLAPW) } \\
\hline & $\begin{array}{l}\left.V_{\tilde{\tilde{\tilde{O}}}}^{(i)} \mathrm{V} / \mathrm{m}^{2}\right] \\
{\left[10^{21}\right.}\end{array}$ & $\eta^{(i)}$ & $\begin{array}{l}\left.V_{\tilde{\tilde{\pi}}}^{(i)} \mathrm{V} / \mathrm{m}^{2}\right] \\
{\left[10^{21} \mathrm{~V} / \mathrm{m}^{2}\right.}\end{array}$ & $\eta^{(i)}$ \\
\hline $\begin{array}{l}1 \\
2\end{array}$ & $\begin{array}{r}18.92(4) \\
4.55(2)\end{array}$ & $\begin{array}{l}0.0 \\
0.053 \text { (3) }\end{array}$ & $\begin{array}{r}12.63 \\
4.90\end{array}$ & $\begin{array}{l}0.0 \\
0.0\end{array}$ \\
\hline
\end{tabular}

out. The vanishing asymmetry parameters at the $\mathrm{W}$ and Re lattice sites are a consequence of the threefold rotation axis.

The presentation of the present results at the "XV International Symposium on Nuclear Quadrupole Interactions" in Leipzig in July 1999 prompted Dr. T. Bastow, CSIRO Manufacturing Science and Technology, Clayton, Australia, to measure the EFG at ${ }^{91} \mathrm{Zr}$ in $\mathrm{ZrW}_{2} \mathrm{O}_{8}$ at $296 \mathrm{~K}$ using high field NMR spectroscopy. The experimental result $\left|V_{z z}^{\mathrm{Zr}}\right|=2.2(1) \cdot 10^{21} \mathrm{~V} / \mathrm{m}^{2}$ is in good agreement with the theoretical result $V_{z z}^{\mathrm{Zr}}=2.45 \cdot 10^{21} \mathrm{~V} / \mathrm{m}^{2}$ shown in Table 2 [19].

\section{Discussion}

All TDPAC spectra of $\mathrm{ZrW}_{2} \mathrm{O}_{8}$ below the phase transition show two characteristic NQIs with almost equal populations which have been identified with the two tungsten sites $\mathrm{W} 1$ and $\mathrm{W} 2$ of the crystal structure of the room temperature phase of $\mathrm{ZrW}_{2} \mathrm{O}_{8}$. This is supported also by the ab-initio EFG calculations at the $\mathrm{W} 1$ and $\mathrm{W} 2$ sites. The non-vanishing inhomogeneous line broadening at both lattice sites even at low temperatures indicates lattice imperfections.

A comparison of the experimental values of the ${ }^{187} \mathrm{Re}$ EFG at the W1 and the W2 site with the values of the impurity calculations is shown in Table 4.

There is good agreement within $8 \%$ for the W2 site. In the case of the W1 site the agreement is within $33 \%$ only. Without more detailed calculations with larger RKM including relaxations and accounting for a possible broken threefold symmetry, we are unable to understand this discrepancy. The calculated asymmetry parameters are a consequence of the threefold rotation axis. Therefore, the experimental non-vanishing asymmetry parameter $\eta^{(2)}$ suggests that the reported crystal structure might be merely an approximation of the real structure, at least for the W2 site. Our previous investigations on tungsten 
compounds which by symmetry should lead to $\eta=0$ [11] indeed exhibited $\eta=0$, thus indicating that the axial symmetry is not broken as a consequence of the nuclear transmutation ${ }^{187} \mathrm{~W} \rightarrow{ }^{187} \mathrm{Re}$.

In the investigated temperature range between $17 \mathrm{~K}$ and $334 \mathrm{~K}$ the quadrupole coupling constant $v_{\mathrm{Q}}^{(1)}$ shows only a small increase of $0.6 \%$, and $v_{\mathrm{Q}}^{(2)}$ remains even constant within the error limits. This agrees well with the assumption of rigid unit modes in the room temperature phase, since the EFG should be essentially constant in the centre of a rigid polyhedron. Only the asymmetry parameter $\eta^{(2)}$ shows a significant thermal variation which appears to parallel the thermal variation of the terminal $\mathrm{W}-\mathrm{O}$ bond length of the W2 tetrahedron (see Figure 10). Therefore, our experiments corroborate the assumption that the origin of the negative thermal expansion of the room temperature structure is a geometric volume contraction due to coupled librations of rigid polyhedra as described in [7].

Both lattice sites show a significant variation of the asymmetry parameter and the line width near the structural phase transition at $428 \mathrm{~K}$. This behaviour reflects the increasing oxygen mobility near the phase transition. No distinct NQI was observed at $503 \mathrm{~K}$ in the high temperature phase, which is probably also due to the high

[1] T. A. Mary, J. S. O. Evans, T. Vogt, and A. W. Sleight, Science 272, 90 (1996).

[2] A. W. Sleight, Annu. Rev. Mater. Sci. 28, 29 (1998).

[3] J. S. O. Evans, T. A. Mary, and A. W. Sleight, J. Solid State Chem. 137, 148 (1998).

[4] P. M. Forster, A. Yokochi, and A. W. Sleight, J. Solid State Chem. 140, 157 (1998).

[5] A. W. Sleight, Curr. Opin. Solid State Mater. Sci. 3, 128 (1998).

[6] J. S. O. Evans, T. A. Mary, T. Vogt, M. A. Subramanian, and A. W. Sleight, Chem. Mater. 8, 2809 (1996).

[7] A. K. A. Pryde, K. D. Hammonds, M. T. Dove, V. Heine, J. D. Gale, and M. C. Warren. J. Phys. Condens. Matter 8, 10973 (1996).

[8] W. Tröger, N. Ulbrich, and T. Butz. Hyperfine Interact. 120/121, 491 (1999).

[9] P. Blaha, K. Schwarz, and J. Luitz. Vienna University of Technology, Vienna 1997. (Improved and updated Unix version of the original copyrighted WIEN-code, which was published by Comput. Phys. Commun. 59, 399(1990)). oxygen mobility and the associated fluctuating occupation of two different $\mathrm{WO}_{4}$ configurations along the threefold axis. It is quite possible that motional narrowing occurs at higher temperatures and a characteristic NQI becomes visible above $503 \mathrm{~K}$. Since there is currently no evidence that rigid unit modes also exist in the high temperature phase, the high oxygen mobility and the resulting alternating occupation of two different $\mathrm{WO}_{4}$ configurations is a possible mechanism of the negative thermal expansion of the high temperature phase. This would also explain why the negative thermal expansion in $\mathrm{ZrW}_{2} \mathrm{O}_{8}$ is not discontinuous at the structural phase transition in contrast to the majority of negative thermal expansion materials.

\section{Acknowledgements}

We are very grateful to U. Wagner and J. E. Punsch, Technical University of Munich as well as D. Alber, Hahn-Meitner-Institut Berlin, for their support in carrying out the irradiations of the samples. It is also a pleasure to thank A. Sleight and J. Gardener for providing the $\mathrm{ZrW}_{2} \mathrm{O}_{8}$ sample. We are grateful to S. C. Bedi, Panjab University, Chandigarh, India, for his support in carrying out the TDPAC-measurements.

[10] H. Haas and D. A. Shirley. J. Chem. Phys. 58, 3339 (1973).

[11] P. Schmidt, T. Soldner, W. Tröger, X. Ni, T. Butz, and P. Blaha. Z. Naturforsch. 53a, 323 (1998).

[12] T. Butz, S. Saibene, Th. Fraenzke, and M. Weber. Nucl. Instrum. Meth. A284, 417 (1989).

[13] T. Butz. Z. Naturforsch. 519, 396 (1996).

[14] J. Graham, A. D. Wadsley, J. H. Weymouth, and L. S. Williams. J. Amer. Ceram. Soc. 42, 570 (1959).

[15] W. Tröger and T. Butz. Z. Naturforsch. 47a, 12 (1992).

[16] T. Butz, M. Ceolín, P. Ganal, P. Schmidt, M. A. Taylor, and W. Tröger. Phys. Scr. 54, 234 (1996).

[17] G. Czjzek. Hyperfine Interact. 14, 189 (1983).

[18] P. Blaha, K. Schwarz, and J. Luitz. Vienna University of Technology 1997.

[19] T. Bastow. Private communication, August 1999. 